\title{
Significant Capacitance Enhancement via In Situ Exfoliation of Quasi-One-Dimensional Graphene Nanostripes in Supercapacitor Electrodes
}

\author{
Jacob D. Bagley, Dorte R. Danielsen, and Nai-Chang Yeh*
}

Cite This: ACS Omega 2021, 6, 5679-5688

Read Online

ACCESS | Lill Metrics \& More | 回 Article Recommendations ｜（s Supporting Information

ABSTRACT: Graphene has received much attention as a supercapacitor electrode material due to its chemical inertness in preventing reaction with electrolytes and the large surface area due to its two-dimensional nature. However, when graphene sheets are processed into electrodes, they tend to stack together and form a turbostratic graphite material with a much reduced surface area relative to the total surface area of individual graphene sheets. Separately, electrochemical exfoliation of graphite is one method of producing single-layer graphene, which is often used to produce graphene for supercapacitor electrodes, although such exfoliated graphene still leads to reduced surface areas due to stacking during electrode fabrication. To utilize the large surface area of graphene, graphene must be exfoliated in situ within a supercapacitor device after the device fabrication. However, graphitic electrodes are typically destroyed upon exfoliation, which is largely due to the loss of electrical connectivity among small exfoliated graphene

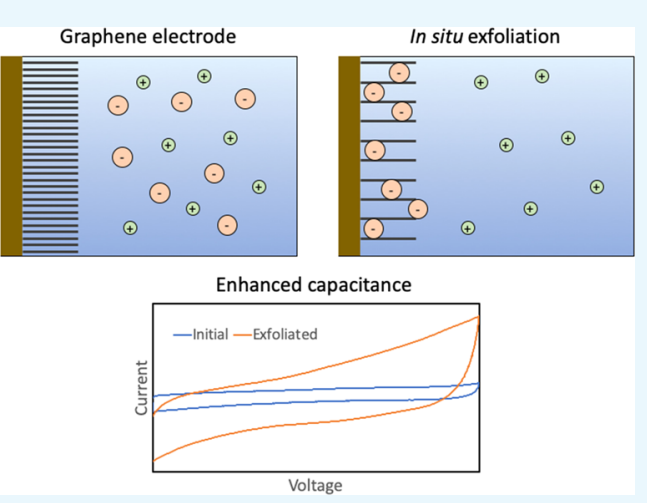
flakes. Here, we report successful in situ exfoliation of graphene nanostripes, a type of quasi-one-dimensional graphene nanomaterial with large length-to-width aspect ratios, as the anode material in supercapacitors. We find that the in situ exfoliation leads to over $400 \%$ enhancement in capacitance as the result of retaining the electrical connectivity among exfoliated quasi-one-dimensional graphene nanostripes in addition to increasing the total surface area, paving ways to fully realizing the benefit of graphene electrodes in supercapacitor applications.

\section{INTRODUCTION}

Extensively studied active materials for supercapacitors include pure graphene, ${ }^{1}$ heteroatom-doped graphene, ${ }^{2,3}$ and graphene composites (e.g., with metal oxide nanoparticles). ${ }^{4}$ Graphene is attractive as a supercapacitor electrode material due to its large surface area as a two-dimensional material and its high carrier mobility and quantum capacitance. ${ }^{5,6}$ An additional advantage is that graphene, as well as other carbon-based nanomaterials, can be deposited on surfaces in precise configurations to make interesting devices such as microsupercapacitors. ${ }^{7}$ However, processing of graphene sheets into supercapacitor electrodes typically leads to stacking of graphene sheets, ${ }^{8}$ reducing the total surface area and decreasing the carrier mobility ${ }^{9}$ such that the advantages of graphene are often not realized in practical supercapacitor devices. Therefore, it is desirable to develop methods to obtain single-layer graphene in practical supercapacitor devices. Indeed, many reports have focused on developing methods to increase the surface area of nanocarbons.

Methods of producing single-layer graphene in high yield include oxidative unzipping of carbon nanotubes, ${ }^{11}$ oxidative chemical exfoliation, ${ }^{12}$ liquid-phase exfoliation, ${ }^{13}$ and electrochemical exfoliation. ${ }^{14}$ Electrochemical exfoliation is the process of separating graphite or multilayer graphene into single-layer graphene sheets by applying a voltage to intercalate large ions, separating graphite sheets to single-layer graphene. This has been done in organic, ${ }^{14}$ aqueous, ${ }^{15}$ and ionic liquid ${ }^{16}$ solutions and has been used to produce graphene for fieldeffect transistors, ${ }^{17}$ energy storage, ${ }^{18}$ transparent conductive electrodes, ${ }^{19}$ and gas sensors. ${ }^{20}$ However, if single-layer graphene is desired for these applications, researchers should be cognizant that packaging the exfoliated graphene into a device typically results in stacking of graphene sheets. ${ }^{8}$ Of these, we find electrochemical exfoliation particularly interesting because it can potentially be applied in situ in a packaged supercapacitor electrode.

Although in situ exfoliation of graphitic electrodes typically catastrophically degrades the electrode performance due to loss of electrical contact as individual graphene sheets separate, ${ }^{21-23}$ we demonstrate in this contribution that by employing quasi-one-dimensional graphene nanostripes

Received: December 16, 2020

Accepted: February 1, 2021

Published: February 18, 2021 
a

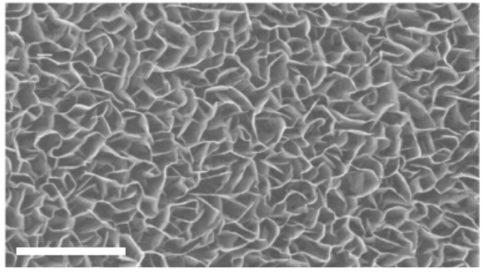

c

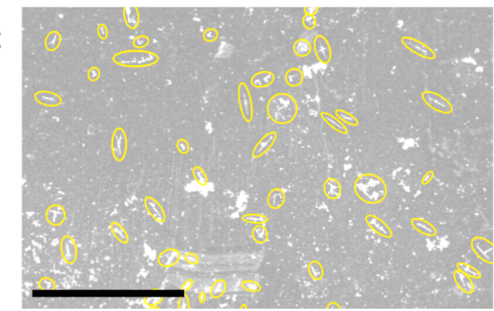

b

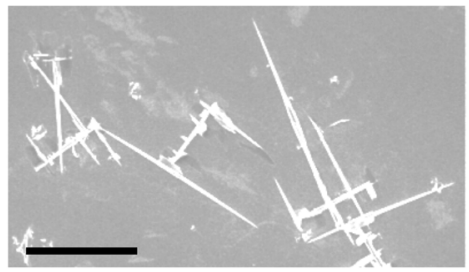

d

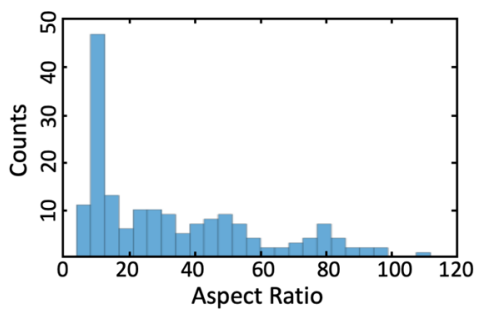

Figure 1. (a) Normal-incidence SEM image of as-grown GNSPs. Scale bar: $8 \mu \mathrm{m}$. (b) SEM image of individual dispersed GNSPs. Scale bar: $16 \mu \mathrm{m}$. (c) Wide-area SEM image of dispersed GNSPs with GNSPs highlighted by yellow circles. Scale bar: $200 \mu \mathrm{m}$. (d) Histogram of aspect ratios of 177 GNSPs.

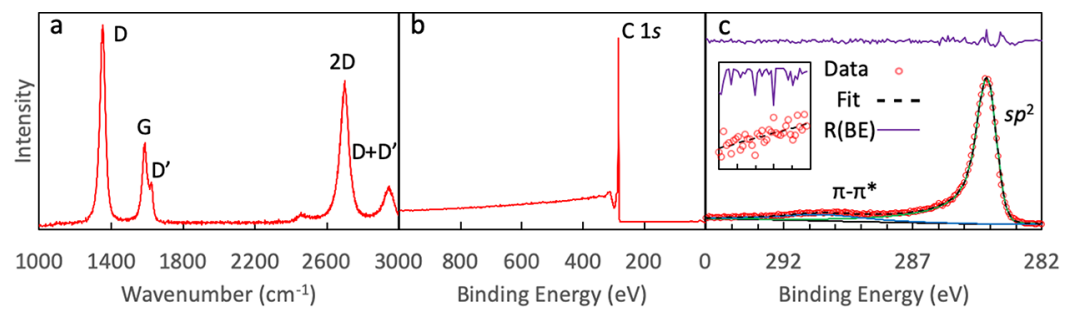

Figure 2. (a) Raman spectrum, (b) XPS survey spectrum, and (c) high-resolution XPS C 1s spectrum of our fabricated GNSPs with fit residuals $\mathrm{R}(\mathrm{BE})$. Inset: Fitting of the section of spectrum outside of the peak area to determine the intrinsic noise of measurement. Here, the binding energy range for the inset is from 292.5 to $295.0 \mathrm{eV}$.

(GNSPs) as the active electrode material, the electrode supercapacitor performance can be enhanced upon in situ exfoliation due to the electrical conductivity maintained by the percolating nature of one-dimensional materials. These concepts, i.e., electrical percolation by quasi-one-dimensional materials and in situ electrochemical modification of electrodes, have actually been explored in other materials ${ }^{24,25}$ but have not been simultaneously studied in carbon nanomaterials to the best of our knowledge. GNSPs are a graphene nanomaterial previously developed in our group that demonstrate chemical purity, good crystallinity, high carrier mobility, and quasi-one-dimensionality (e.g., GNSP dimensions are $\sim 400 \mathrm{~nm} \times 60 \mu \mathrm{m}){ }^{26}$ In our study of in situ exfoliated GNSPs, we employ X-ray diffraction (XRD) to study structural changes in the material and electrochemical impedance spectroscopy (EIS) to study the behavior of the electrode at various degrees of exfoliation and conjecture that the improved capacitance is due to an increase in surface area while maintaining the electrical connectivity in the electrode.

\section{RESULTS}

The quasi-one-dimensional nature of GNSPs is demonstrated in Figure 1. Figure 1a is a normal-incidence scanning electron microscopy (SEM) image of GNSPs on the growth substrate without further modification. Here, the one-dimensional nature is not apparent, but the fabricated material can be seen as a vertically oriented graphene material, where graphene sheets grow vertically with respect to the growth substrate and form a dense interconnected network. Detailed reviews of vertically oriented graphene can be found in refs 28,29 . When dispersed onto a substrate (Figure 1b), our GNSPs, which are a particular class of vertically oriented graphene nanomaterial, become apparently quasi-one-dimensional with large length-towidth aspect ratios. Several GNSPs are visible in Figure 1b, but a wider area image (Figure 1c) shows that the GNSP dispersion contains a large amount of GNSPs (highlighted in Figure 1c) with varying dimensions. An analysis of 177 individual GNSPs reveals that the average width and length of GNSPs are $\sim 450 \mathrm{~nm}$ and $\sim 12 \mu \mathrm{m}$, respectively. A histogram of analyzed GNSPs aspect ratios is provided in Figure 1d, where the average aspect ratio is $\sim 34: 1$, and the most frequent aspect ratio is $\sim 10: 1$. (Note: these GNSPs do not exhibit quantum confinement effects as their typical width is $\sim 450$ $\mathrm{nm}$ while quantum confinement effects begin at widths below $\sim 40 \mathrm{~nm}^{30}$ ) The Brunauer-Emmett-Teller (BET) surface area was $64 \mathrm{~m}^{2} / \mathrm{g}$.

The successful fabrication of a graphene material was confirmed by Raman spectroscopy and X-ray photoelectron spectroscopy (XPS). The Raman spectrum is presented in Figure 2a. The D $\left(1361 \mathrm{~cm}^{-1}\right), \mathrm{G}\left(1589 \mathrm{~cm}^{-1}\right)$, and 2D (2704 $\mathrm{cm}^{-1}$ ) peaks are characteristic of graphene, and the $\mathrm{D}^{\prime}(1609$ $\left.\mathrm{cm}^{-1}\right)$ and $\mathrm{D}+\mathrm{D}^{\prime}\left(2945 \mathrm{~cm}^{-1}\right)$ peaks together with the $\mathrm{G}$ and $\mathrm{D}^{\prime}$ peaks being well resolved classify the sample as a crystalline graphene nanomaterial according to the three-stage defect model. $^{31,32} \mathrm{We}$ also acknowledge that the $\mathrm{D}$ peak is very intense in this nanomaterial, which may be partially due to the abundant edges.

The XPS survey spectrum (Figure 2b) demonstrates the chemical purity of the graphene material, as the only visible peak (at $\sim 285 \mathrm{eV}$ ) corresponds to $\mathrm{C} 1 \mathrm{~s}$. To further investigate 

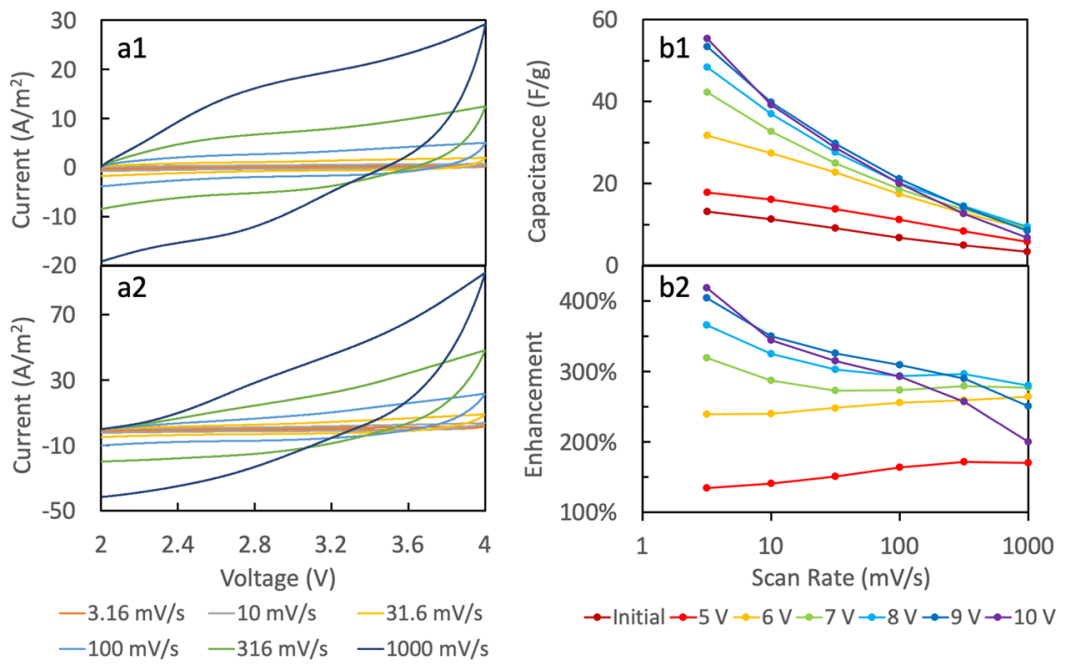

Figure 3. (a1) Cyclic voltammograms of a preexfoliated GNSP electrode. (a2) Cyclic voltammograms of a GNSP electrode exfoliated at 10 V. (b1) Specific capacitance of each electrode as a function of scan rate. Here, the "initial" curve denotes the capacitance of preexfoliated GNSP electrode. (b2) Capacitive enhancement of each exfoliated GNSP electrode with respect to the preexfoliated GNSP electrode as a function of scan rate.

the nature of the carbon in the material, we studied the $\mathrm{C} 1 \mathrm{~s}$ peak by a high-resolution narrow scan (Figure 2c). We fit the $\mathrm{C} 1 \mathrm{~s}$ spectrum with two components corresponding to $\mathrm{sp}^{2}$ hybridized C-C bonds $(284.1 \mathrm{eV})$ and a $\pi-\pi^{*}$ satellite $(290.5$ $\mathrm{eV}$ ) with a Tougaard background. The $\mathrm{sp}^{2}$ peak was fit to a finite Lorentzian line shape with an asymmetry parameter of 0.18 and a full-width at half-maximum of $0.87 \mathrm{eV}$, consistent with previous reports. ${ }^{33,34}$ Here, the finite Lorentzian line shape refers to a convolution of the Lorentzian and Gaussian functions, with the Lorentzian function asymmetrically raised to different exponentials for binding energies above and below the peak center. This approach therefore creates asymmetric tails, which is useful for modeling conducting materials. We further note that the finite Lorentzian line shape is an established method for modeling the $\mathrm{C} 1 \mathrm{~s} \mathrm{sp}^{2}$ component, whose use and practical applications have been discussed in various sources, ${ }^{35-37}$ and that the specific function used for this spectral analysis was defined as $\operatorname{LF}(0.53,1.2,240,250,3)$ in the CasaXPS software. The $\pi-\pi^{*}$ satellite was fit with a symmetric Gaussian-Lorentzian function with a width of 3.6 $\mathrm{eV}$. Given that the $\mathrm{sp}^{2}$ peak and the satellite were fairly well resolved, we did not impose constraints on the peak widths and peak positions. The fitted peak positions (i.e., the energy separation of $6.4 \mathrm{eV}$ between the $\mathrm{sp}^{2}$ and the satellite peaks ${ }^{38}$ ) as well as the width of the $\mathrm{sp}^{2}$ peak thus obtained were found to be consistent with the literature, which confirmed the validity of our fit. The residuals spectrum in Figure $2 c$ further demonstrated that there was no need to consider additional peaks in the fitting. The fit yielded an Abbe criterion of 0.67, which was not ideal compared with the Abbe criterion of 1 for perfect noise around a fit. However, a linear fit to the spectral background (Figure 2c, inset) gave an Abbe criterion of 0.69, suggesting that the Abbe criterion of the peak fit was reasonable based on the intrinsic noise in this spectrum. ${ }^{39}$

The strong $\mathrm{sp}^{2}$ peak and the presence of the $\pi-\pi^{*}$ peak verify the graphene crystallinity, i.e., domains with delocalized p-orbitals. ${ }^{34,40}$ The combined results of Raman spectra and XPS data confirmed that the GNSPs are highly crystalline and chemically pure graphene material. Further characterization of the GNSPs, including transmission electron microscopy, energy-dispersive $\mathrm{X}$-ray spectroscopy, selected area electron diffraction, and ultraviolet photoelectron spectroscopy can be found in ref 26.

The GNSPs were packaged into a supercapacitor in a coin cell configuration, and the capacitance was measured by cyclic voltammetry at various scan rates between 2 and $4 \mathrm{~V}$, as shown in Figure 3a1. The cyclic voltammograms were nearly rectangular, indicating a fairly ideal electrochemical doublelayer capacitor process with little contribution from redox processes. ${ }^{41}$ The nonidealities, such as the positive slope while charging and negative slope while discharging, may arise from slight changes in the intercalation state of $\mathrm{Li}^{+}$or $\mathrm{PF}_{6}^{-}$(e.g., $\mathrm{Li}^{+}$ intercalation could occur at voltages as high as $2.5 \mathrm{~V}$ in disordered graphene materials; for further discussion on the matter, see Supporting Information Note 1), which had also been reported previously in graphene supercapacitors. ${ }^{1}$ In addition, these electrodes demonstrated the classic capacitor behavior of increasing current response with increasing scan rate. $^{41}$

Graphene electrodes were electrochemically exfoliated in situ by ramping the cell voltage to $1 \mathrm{~V}$, holding the cell voltage at $1 \mathrm{~V}$ for $3 \mathrm{~h}$, then ramping the cell voltage to a high voltage, holding the cell voltage at the high voltage for $3 \mathrm{~h}$, then ramping down to $2 \mathrm{~V}$. The high voltages ranged from 5 to 10 V. The rationale for this voltage program was that a high voltage caused intercalation of $\mathrm{PF}_{6}{ }^{-21,44}$ which, as a large molecule, induced separation of graphene sheets (i.e., exfoliation), and the low voltage $(1 \mathrm{~V})$ caused intercalation of a $\mathrm{Li}^{+}$-propylene carbonate complex, which also exfoliated the graphene. ${ }^{22} \mathrm{We}$ also performed these experiments without the $1 \mathrm{~V}$ exfoliation step, and the capacitance of these electrodes did not enhance as well as those that included the $1 \mathrm{~V}$ exfoliation step (see Figure S2).

Märkle et al. ${ }^{21}$ performed a similar exfoliation procedure using the same electrolyte ( $1 \mathrm{M} \mathrm{LiPF}_{6}$ in propylene carbonate/ dimethyl carbonate), the same counter/reference electrode (lithium metal), and similar cycling between relatively low (3 $\mathrm{V}$ ) and high voltages $(5.5 \mathrm{~V})$ except employing a regular (not quasi-one-dimensional) graphitic material in the working electrode, but observed "[e]lectrical contact loss between the graphite particles themselves and/or particles and the current collector". The only significant difference between the system 


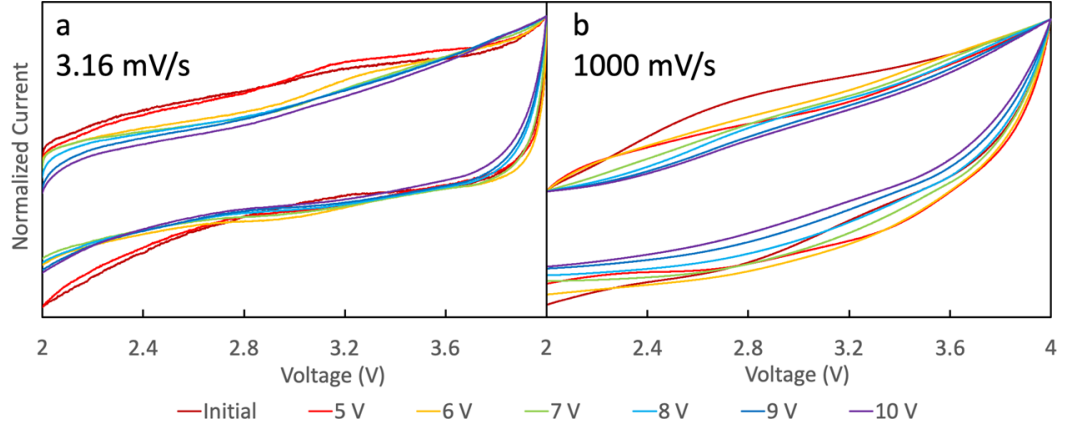

Figure 4. Normalized current response for each electrode at $3.16 \mathrm{mV} / \mathrm{s}$ (a) and $1000 \mathrm{mV} / \mathrm{s}(\mathrm{b})$.

described in this contribution and the system studied by Märkle et al. is that we are employing quasi-one-dimensional GNSPs while they did not, and, as demonstrated herein, instead of observing catastrophic device failure, we observe significantly enhanced capacitance due to exfoliation.

Before proceeding further, we remark that using high exfoliation voltages may initially seem concerning since the stability window of the electrolyte in this configuration is reported as only $6 \mathrm{~V} \cdot{ }^{45,46}$ Indeed, we conjecture below that this high voltage may cause some decomposition that affects ion transport. Further, such high voltages may be dangerous. However, in our experiments, we did not observe damages or danger on a macroscopic scale, and the microscopic damages, although present, were slight. We believe that these findings were due to the relatively small voltage gradient at any point within the cell despite the fact that the total voltage across the entire coin cell may be large. This behavior is typical of supercapacitors, as ions migrate to biased electrodes and screen the total voltage, ${ }^{47}$ and ion intercalation into the bulk of the GNSPs electrode may also provide additional screening.

After exfoliation, the capacitance was measured again by cyclic voltammetry under the same conditions. The cyclic voltammograms for graphene exfoliated at $10 \mathrm{~V}$ are shown in Figure 3a2. The voltammograms of the electrode exfoliated at $10 \mathrm{~V}$ (Figure 3a2) revealed two differences from the preexfoliated graphene (Figure 3a1): (i) the current at a given voltage was much higher, demonstrating a higher capacitance, and (ii) the nonideal behavior, i.e., the slope, was more uniform through the voltammogram. The latter phenomenon may be due to intercalation behavior through the entire voltage range, which may be made possible by an increase in interlayer spacing and a decrease in intercalation potential. The cyclic voltammograms for graphene electrodes exfoliated at 5, 6, 7, 8, and $9 \mathrm{~V}$ as well as the reduced-scale cyclic voltammograms (to visualize lower scan rates) of all electrodes are provided in Figures S2-S9.

We calculated the specific capacitance, $C$, of the electrode at each degree of exfoliation (the preexfoliation capacitance and the capacitance after each exfoliation step) according to eq 1

$$
C=\frac{\int I \mathrm{~d} V}{2 \nu m \Delta V}
$$

where $\int I \mathrm{~d} V$ is the integrated area of the current response $(I)$ of the cyclic voltammetry curve with the voltage differential $(\mathrm{d} V), \nu$ is the scan rate, $m$ is the mass of the graphene, and $\Delta V$ is the voltage window of the cyclic voltammetry scan. The capacitances are shown in Figure $3 \mathrm{~b}$. These data follow the classic supercapacitor behavior of decreasing capacitance with increasing scan rate. ${ }^{41}$ For the lowest scan rate, the capacitance increased steadily with increasing exfoliating voltage. For the highest scan rate; however, exfoliation at $8 \mathrm{~V}$ resulted in the best performance, while exfoliation at 9 and $10 \mathrm{~V}$ resulted in lower capacitance for high scan rates. This decrease in performance for exfoliation at 9 and $10 \mathrm{~V}$ may be due to solvent decomposition on the electrode surface during exfoliation, ${ }^{4,46}$ producing byproducts that impaired ion transport to and from the electrode such that the capacitance was impaired for high scan rates but not low scan rates.

To quantitatively compare the performance of each exfoliated electrode with respect to the preexfoliated electrode, we calculated the capacitive enhancement at each scan rate $\left(E_{\nu}\right)$ according to eq 2

$$
E_{\nu}=\frac{C_{\text {exfoliated }, \nu}}{C_{\text {initial }, \nu}} \times 100 \%
$$

where $C_{\text {exfoliated, }, \nu}$ is the specific capacitance at a scan rate, $\nu$, of the exfoliated graphene and $C_{\text {initial }, \nu}$ is the specific capacitance at a scan rate, $\nu$, of the preexfoliated graphene. Figure $3 \mathrm{~b} 2$ plots the capacitive enhancement at each scan rate and demonstrates an enhancement of $418 \%$ for graphene exfoliated at $10 \mathrm{~V}$. In addition, for graphene electrodes exfoliated at 5 and $6 \mathrm{~V}$, the enhancement increases with increasing scan rate, whereas for graphene electrodes exfoliated at 9 and $10 \mathrm{~V}$, the enhancement decreases with increasing scan rate. For graphene electrodes exfoliated at 7 and $8 \mathrm{~V}$, the enhancement first decreases and then increases with increasing scan rate.

To further understand this increase in capacitance, we investigated whether its origin was associated with an increase in nonfaradic charge storage (i.e., double-layer capacitance) or in faradic charge storage (i.e., redox reactions). An increase in the double-layer capacitance would result from an increase in the effective surface area, while an increase in faradic charge storage would result from chemical activation of the graphene material such that redox-active sites were created. Nonfaradic and faradic processes can be distinguished by comparing the cyclic voltammograms with fast and slow scan rates. Generally, nonfaradic (capacitive) current dominates at fast scan rates, and faradic current dominates at slow scan rates. Therefore, if a faradic process is taking place at an electrode, then a redox peak at some voltage will be visible at low scan rates but will be hidden by the nonfaradic current at high scan rates. ${ }^{47}$ To determine whether the increased capacitance observed here involved faradic processes, we normalized the cyclic voltammogram current response for each exfoliation step at the slowest scan rate (Figure $4 a$ ) and the highest scan rate (Figure $4 b$ ). In both plots, we observe that the current curves were similar and quasi-rectangular for all electrodes exfoliated at 6-10 V for the slowest scan rate and at $5-10 \mathrm{~V}$ at the highest scan rate, 
suggesting that the electrochemical processes did not change significantly with exfoliation. For further discussion of the evidence for the increase of surface area during exfoliation, see Supporting Information Note 2.

Next, we investigated the effect of the number of exfoliation cycles on capacitance enhancement. For these experiments, the capacitance was measured then the GNSPs electrode was held at $1 \mathrm{~V}$ for $1 \mathrm{~h}$, then at $6 \mathrm{~V}$ for $1 \mathrm{~h}$, then returned to $2 \mathrm{~V}$ for a cyclic voltammogram capacitance measurement. This process was repeated for 20 cycles. Similar measurements were also repeated using $10 \mathrm{~V}$ instead of $6 \mathrm{~V}$. These voltages (6 and 10 $\mathrm{V})$ were chosen because $6 \mathrm{~V}$ was the electrochemical stability window of the electrolyte, ${ }^{45,46}$ and previous measurements demonstrated the greatest enhancement at $10 \mathrm{~V}$. The cycledependent exfoliation using a high voltage of 6 and $10 \mathrm{~V}$ is shown in Figure 5. In both cases, exfoliation always enhanced

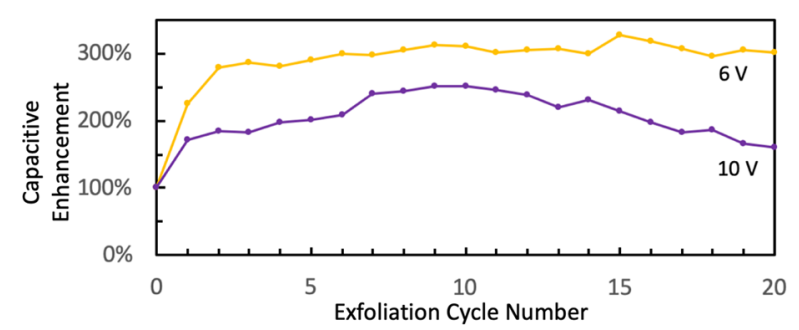

Figure 5. Cycle-dependent exfoliation capacitance enhancement using high voltages of 6 and $10 \mathrm{~V}$.

the capacitance, but the number of cycles for maximum enhancement differed: For exfoliation at $6 \mathrm{~V}$, the capacitance enhancement plateaued after two cycles and resulted in $~ 300 \%$ capacitance enhancement, whereas for exfoliation at $10 \mathrm{~V}$, enhancement maximized at $\sim 250 \%$ after nine cycles, then decayed with increasing cycles.

The data shown in Figure 5 contrast with the results presented in Figure 3 in two aspects: In Figure 3, exfoliation at $10 \mathrm{~V}$ resulted in higher capacitance than exfoliation at $6 \mathrm{~V}$; and exfoliation at $10 \mathrm{~V}$ resulted in $\sim 420 \%$ capacitance enhancement in Figure 3 rather than $\sim 250 \%$ enhancement in Figure 5. We resolve this discrepancy by considering that the electrolyte was not stable under a voltage gradient of $10 \mathrm{~V}^{45,46}$ so that exfoliating at $10 \mathrm{~V}$ may have caused solvent decomposition that was destructive to the capacitance. In addition, exfoliating at lower voltages before exfoliating at $10 \mathrm{~V}$ may enable better ion transport into the bulk of the electrode (e.g., via pores) that helped screen the total voltage such that when a voltage was applied to the electrode, there was a voltage gradient across the electrode, and the voltage gradient at the solutionelectrode interface was not too intense. In other words, if the electrode was partially exfoliated before applying a high voltage, then counter ions in the bulk of the electrode would help screen the voltage for the electrode-solution interface when a high voltage was applied, leading to reduced electrolyte decomposition while enabling further exfoliation.

To better understand the cause of increased capacitance in exfoliated electrodes, we measured the X-ray diffraction (XRD) patterns of our GNSP electrodes exfoliated at different voltages (Figure 6). Specifically, we investigated the XRD spectra of an as-grown GNSP electrode, a GNSP electrode exfoliated at $6 \mathrm{~V}$ for 20 cycles, and a GNSP electrode exfoliated at $10 \mathrm{~V}$ for 20 cycles. Interestingly, for both the as-grown electrode and the electrode exfoliated at $6 \mathrm{~V}$, the position of the [002] peak was

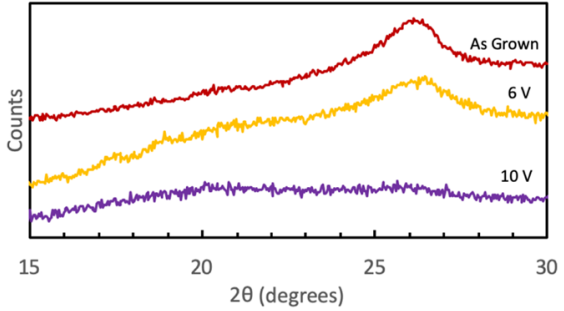

Figure 6. XRD pattern near the [002] peak for as-grown graphene, graphene exfoliated at $6 \mathrm{~V}$, and graphene exfoliated at $10 \mathrm{~V}$.

similar, but for the electrode exfoliated at $10 \mathrm{~V}$, the [002] peak disappeared. According to Bragg's law $\left(d_{002}=n \lambda / 2 \sin \theta\right.$, where $n$ is a positive integer, $\lambda$ is the incident X-ray wavelength, and $\theta$ is the diffraction angle), the interlayer spacings in the as-grown graphene and graphene exfoliated at $6 \mathrm{~V}$ were 3.40 and $3.37 \AA$, respectively. Given that the [002] peak indicated the interlayer spacing, the presence of the [002] peak implied that applying a voltage of $6 \mathrm{~V}$ did not fully exfoliate the graphene (i.e., separate it into individual monolayers) even though the peak intensity was reduced and linewidth broadened (suggesting partial exfoliation). In contrast, applying a voltage of $10 \mathrm{~V}$ fully exfoliated the GNSP electrode so that there was no longer ordered $c$-axis stacking, hence the complete disappearance of the [002] peak.

Finally, we used electrochemical impedance spectroscopy (EIS) to further understand the changes taking place during the exfoliation that led to increased capacitance. The Nyquist plots for each exfoliation step are shown in Figure 7a. The impedance data were fit to a modified Randles circuit (Figure $7 \mathrm{~b}$ ) with the fit parameters and mean-square errors (MSE) provided in Table 1 . In the equivalent circuit, the capacitor and the Warburg diffusion elements are replaced with constant phase elements $\left(\mathrm{CPE}_{1}\right.$ and $\mathrm{CPE}_{\mathrm{W}}$, respectively) to account for the nonidealities.

Before interpreting the equivalent circuits and the model parameters, we introduce the mathematical relations of the circuit elements. The impedances of a capacitor $\left(Z_{\mathrm{C}}\right)$, Warburg element $\left(Z_{\mathrm{W}}\right)$, and constant phase element $\left(Z_{\mathrm{CPE}}\right)$ are defined, respectively, in eqs $3-5$

$$
\begin{aligned}
& Z_{\mathrm{C}}=\frac{1}{\mathrm{i} \omega C} \\
& Z_{\mathrm{W}}=\frac{1}{Q \sqrt{\mathrm{i} \omega}} \\
& Z_{\mathrm{CPE}}=\frac{1}{Q(\mathrm{i} \omega)^{n}}
\end{aligned}
$$

where $\mathrm{i}$ is the imaginary number, $\omega$ is the radial frequency, $C$ is the capacitance, $R$ is the resistance, and $n$ and $Q$ are constants. $^{41-43}$ In the cases of $n=1$ and 0.5 , the constant phase element (CPE) becomes mathematically identical to the capacitor and Warburg element, respectively. Therefore, a CPE is often used to model nonideal capacitors and Warburg elements. ${ }^{41,48}$ For a CPE that is modeling a capacitor, the extent to which $n$ deviates below 1 can be interpreted as the "leakiness" of the capacitor, and for a CPE modeling a Warburg element, values of $n$ deviating above 0.5 indicate diffusion within a porous three-dimensional structure (as opposed to diffusion near a perfect two-dimensional plane). ${ }^{41,48,49}$ 


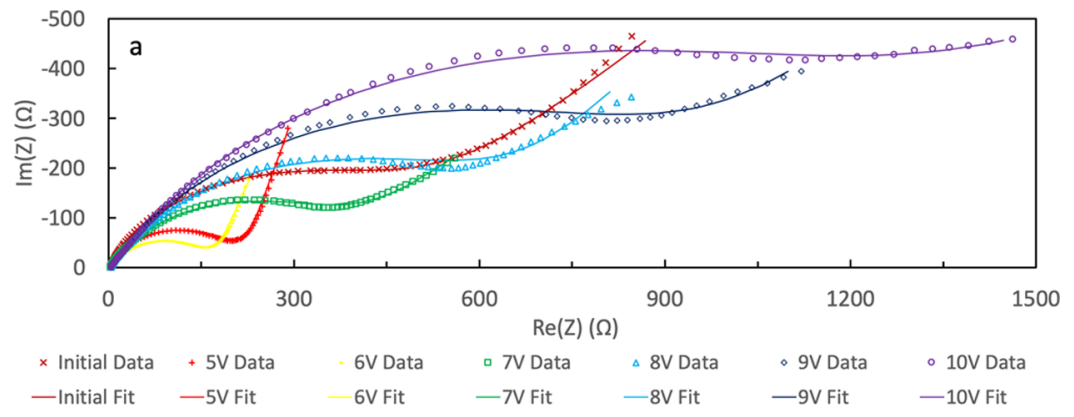

b

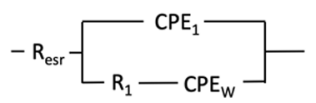

Figure 7. (a) Nyquist plots for electrode exfoliated at the indicated high voltage in the range of $0.1 \mathrm{MHz}$ to $1 \mathrm{~Hz}$. (b) Equivalent circuit model for the Nyquist plots data.

Table 1. Model Fitting Parameters of the Data in Figure 7a According to the Circuit in Figure $7 \mathrm{~b}$

\begin{tabular}{|c|c|c|c|c|c|c|c|}
\hline & initial & $5 \mathrm{~V}$ & $6 \mathrm{~V}$ & $7 \mathrm{~V}$ & $8 \mathrm{~V}$ & $9 \mathrm{~V}$ & $10 \mathrm{~V}$ \\
\hline$R_{\mathrm{esr}}(\Omega)$ & 0.6 & 2.3 & 2.1 & 4.6 & 1.5 & 1.9 & 4.8 \\
\hline$R_{1}(\Omega)$ & 509 & 226 & 178 & 332 & 694 & 1001 & 1254 \\
\hline$n_{1}$ & 0.74 & 0.74 & 0.68 & 0.75 & 0.67 & 0.67 & 0.68 \\
\hline$Q_{1}\left(S s^{\wedge} n_{1}\right)$ & $1.7 \times 10^{-5}$ & $2.5 \times 10^{-5}$ & $4.4 \times 10^{-5}$ & $2.2 \times 10^{-5}$ & $3.8 \times 10^{-5}$ & $3.4 \times 10^{-5}$ & $2.9 \times 10^{-5}$ \\
\hline$n_{\mathrm{W}}$ & 0.51 & 0.83 & 0.80 & 0.42 & 0.62 & 0.57 & 0.42 \\
\hline$Q_{\mathrm{W}}\left(\mathrm{S} \mathrm{s}^{\wedge} n_{\mathrm{W}}\right)$ & $6.4 \times 10^{-4}$ & $7.4 \times 10^{-4}$ & $1.2 \times 10^{-3}$ & $1.4 \times 10^{-3}$ & $8.3 \times 10^{-4}$ & $8.6 \times 10^{-4}$ & $9.0 \times 10^{-4}$ \\
\hline MSE & 17.2 & 3.3 & 2.4 & 10.8 & 67.3 & 56.7 & 37.7 \\
\hline
\end{tabular}

We provide the following interpretation of the modeling parameters shown in Figure $7 \mathrm{~b}$. In the equivalent circuit, $\mathrm{CPE}_{1}$ represents the double-layer capacitance, $R_{1}$ represents intercalation charge transfer resistance, and $\mathrm{CPE}_{\mathrm{W}}$ represents diffusion behavior. In addition, the "leakage" from $\mathrm{CPE}_{1}$ may represent some intercalation behavior.

Exfoliating to 5 and $6 \mathrm{~V}$ increased $Q_{1}$ (which is often interpreted as the capacitance ${ }^{41}$ ), decreased the intercalation resistance $\left(R_{1}\right)$, and increased $n_{\mathrm{W}}$ (implying three-dimensional diffusion behavior). We physically interpret these parameter changes as an increase in the surface area and decrease in the intercalation energy barrier due to better electrolyte contact and an increase in porosity of the material, respectively. Exfoliating at or above $7 \mathrm{~V}$ nearly restored the diffusion behavior to a Warburg element, which we interpret as the material becoming sufficiently exfoliated such that pores were large enough to behave quasi two-dimensionally.

For exfoliating voltages above $7 \mathrm{~V}, R_{1}$ increased substantially with increasing exfoliating voltage. This may be due to electrolyte decomposition, which impeded ion transport ${ }^{45,46}$ and likely responsible for the degraded performance at high scan rates for graphene electrodes exfoliated above $7 \mathrm{~V}$ (see Figure 3 ). In addition, $n_{1}$ decreased to 0.67 , indicating a very leaky capacitor. It is interesting, however, that these electrodes demonstrated an increased capacitance (at low scan rates, see Figure 3) despite electrolyte decomposition. On the other hand, for exfoliating voltages above $7 \mathrm{~V}$, the MSE of the fit increased substantially, indicating that the device no longer complied with a Randles circuit model, which may be attributed to nonidealities caused by electrolyte decomposition.

\section{DISCUSSION}

Our capacitance measurements demonstrate that GNSP electrodes can be exfoliated in situ to substantially enhance their capacitance (Figure 3), which is made possible by the quasi-one-dimensional nature of GNSPs (Figure 1). Comparison of the current responses of each electrode at both fast and slow scan rates indicates that the charge storage processes in the GNSP electrodes are nonfaradic (Figure 4), which suggests that the increase in capacitance is due to structural rather than chemical changes (e.g., chemical functionalization). Comparing the cycle-dependent exfoliated GNSP electrode (Figure 5) with the gradually exfoliated GNSP electrode (Figure 3) demonstrates that when exfoliating at $10 \mathrm{~V}$, initial exfoliation at a lower voltage results in better capacitance. Our XRD data (Figure 6) reveal that an applied voltage of $6 \mathrm{~V}$ does not fully exfoliate the GNSP electrode, whereas applying a voltage of 10 $\mathrm{V}$ completely exfoliates the electrode. Finally, our EIS data (Figure 7) suggest that the electrochemical processes have fundamentally altered with exfoliation: the diffusion behavior first shifts from a two-dimensional to a three-dimensional diffusion behavior, suggesting that the material becomes porous upon exfoliation. In addition, the double-layer capacitance increases and the intercalation resistance decreases, suggesting better electrolyte contact with the material. As the material becomes sufficiently exfoliated, the diffusion returns to a two-dimensional behavior. The EIS data also suggest that some intercalation charge transfer has taken place. However, noting that the electrochemical processes are nonfaradic based on the cyclic voltammetry data, the intercalation charge transfer is likely minimal, although it may account for the observation of nonideal capacitive charge storage.

We acknowledge that our reported capacitances $(55 \mathrm{~F} / \mathrm{g}$, see Figure $3 \mathrm{~b} 1$ ) are not particularly good, even for the best- 
performing devices (Liu et al., ${ }^{1}$ for example, report a capacitance of $154 \mathrm{~F} / \mathrm{g}$ for a graphene electrode). However, obtaining a high capacitance was not the purpose of this study per se; rather, the purpose of this study was to determine whether graphene electrodes could be exfoliated in situ to enhance their capacitance. To meet this purpose, we designed experimental conditions to probe this phenomenon rather than obtain a high capacitance. For example, our cyclic voltammetry window was $2-4 \mathrm{~V}$, whereas it could have been $1-6 \mathrm{~V}$ (the stability window of the electrolyte), which would have increased the measured capacitance but would have also affected the exfoliation. In addition, our choice of electrolyte ( $\mathrm{LiPF}_{6} /$ propylene carbonate/dimethyl carbonate) may not have resulted in the highest capacitance (e.g., previous studies have shown higher capacitance in aqueous electrolytes ${ }^{1}$ ), but we chose this electrolyte because exfoliation of graphene/ graphite in $\mathrm{LiPF}_{6} /$ propylene carbonate solutions is been a wellstudied phenomenon. ${ }^{1421-23}$ Noting that electrochemical exfoliation of graphite has been demonstrated in other organic solvents, aqueous solvents, and ionic liquids, ${ }^{14-16}$ similar procedures could be applied to other supercapacitor systems with one-dimensional graphene electrodes to optimize the enhancement of capacitance. In addition, quasi-one-dimensional graphene electrodes for supercapacitors could be exfoliated prior to being assembled in a final device to further improve on capacitance.

\section{CONCLUSIONS}

We have demonstrated, in this work, a significant increase in the capacitance of quasi-one-dimensional graphene electrodes by in situ electrochemical exfoliation and provided experimental evidence for a substantial increase in the total surface area of the graphene electrode after exfoliation. This development overcomes the typical restacking problems of graphene electrodes that suffer a significant reduction of the effective surface area and affect the advantages of using graphene electrodes in supercapacitors. Through in situ electrochemical exfoliation, we observe more than $400 \%$ capacitance enhancement relative to the control samples. Thus, the method of in situ electrochemical exfoliation of quasi-one-dimensional graphene nanostripes as electrodes together with further optimized electrolyte solutions and/or exfoliation protocols is expected to be a promising approach toward fully realizing the benefits of graphene as superior supercapacitor electrode material.

While the developments in this work are encouraging, practical implementation of this in situ exfoliation procedure will require further research due to the low specific capacitance and uncertainty in the long-term performance of in situ exfoliated GNSPs supercapacitor electrodes. In particular, the long-term cycling stability of in situ exfoliated GNSPs will be a subject of future studies. Although long-term cycling is typically a routine analysis for supercapacitor electrodes, in this case, a study of long-term cycling is a time-intensive multidimensional endeavor because the cycling stability will likely be influenced by the exfoliating voltage, the voltage range during cycling, and the electrolyte solution.

Future studies will also investigate improving the specific capacitance of in situ exfoliated supercapacitor electrodes. For example, we are interested in mixing GNSPs with high capacitance carbon materials (e.g., 2\% GNSPs in activated carbon) and performing the exfoliation procedure, which may improve the surface area of the activated carbon while maintaining the electrical connectivity with GNSPs. We are also interested in using exfoliated GNSPs to form percolating networks among high capacitance metal oxide nanoparticles. Overall, we consider in situ exfoliation of electrode materials together with the incorporation of quasi-one-dimensional GNSPs a promising research direction for achieving substantially improved supercapacitors.

\section{EXPERIMENTAL SECTION}

GNSP Fabrication. GNSPs were fabricated by plasmaenhanced chemical vapor deposition (PECVD) described previously. ${ }^{26,27}$ Specifically, a microwave-induced hydrogen/ methane plasma with trace 1,2-dichlorobenzene (Alfa Aesar, $99 \%)$ yielded GNSPs on a $\sim(0.75 \mathrm{~cm} \times 1.25 \mathrm{~cm})$ copper foil (McMaster-Carr, $99.9 \%$ ) in a $1 / 2^{\prime \prime}$ outer diameter quartz tube. The PECVD growth system was custom built and consisted of eight parallel deposition chambers each fitted with an Evenson Cavity (Opthos Instruments Inc., Frederick, MD) excited by $70 \mathrm{~W}$ of $2.45 \mathrm{GHz}$ microwave power source (ENS $4 \times 200 \mathrm{~W}$ CPS, Sairem, Décines-Charpieu, France). The plasma volume was $\sim 1 \mathrm{~cm}^{3}$. $\mathrm{H}_{2}$ (MATHESON, 99.999\%) and $\mathrm{CH}_{4}$ (MATHESON, 99.999\%) gases were introduced to the chambers by mass flow controllers (MC series, Alicat Scientific, Tuscon, AZ), and 1,2-dichlorobenzene was placed in a vacuum-sealed vial and introduced to the chamber through a leak valve. The pressure in the chamber before splitting into eight chambers was held at 4.8 Torr, the total flow rates of $\mathrm{H}_{2}$ and $\mathrm{CH}_{4}$ were 48 and $5 \mathrm{sccm}$, respectively, and the ratio of $\mathrm{CH}_{4}$ to 1,2-dichlorobenzene (3-chloropyridine) was $\sim 2: 1$ as measured by a residual gas analyzer (RGA; XT300M, Extorr Inc., New Kensington, PA) placed upstream of the deposition chamber and connected via a capillary. The plasma was maintained for $\sim 3 \mathrm{~h}$ to synthesize sufficient graphene material for use as a supercapacitor electrode.

GNSP Characterization. The resulting GNSP material was characterized by scanning electron microscopy (SEM), Brunauer-Emmett-Teller (BET) surface area method, Raman spectroscopy, X-ray photoelectron spectroscopy (XPS), and X-ray diffraction (XRD). SEM was performed using a Hitachi S-4100 (Hitachi, Tokyo Japan) with an accelerating voltage of $5 \mathrm{kV}$. Individual GNSPs were obtained for imaging by sonicating the copper growth substrate with GNSPs grown on it in $N$-methyl-2-pyrrolidinone (NMP, Sigma-Aldrich, $99.5 \%)^{13}$ for $30 \mathrm{~min}$, centrifuging the dispersion to remove undispersed chunks of graphitic material at $850 \mathrm{rpm}$ for $90 \mathrm{~min}$, extracting one drop of dispersion, and evaporating it over a silicon wafer in low vacuum at $70{ }^{\circ} \mathrm{C}$. The BET surface area was measured in a BELSORP-max volumetric instrument (BEL-Japan, Inc.) via equilibrium $\mathrm{N}_{2}$ adsorption isotherm at $77 \mathrm{~K}$. Raman spectroscopy was performed in a Renishaw M-1000 Micro-Raman (Renishaw, Gloucestershire, U.K.) spectrometer operating with a $514.5 \mathrm{~nm}$ argon-ion laser with a spectral resolution of $1 \mathrm{~cm}^{-1}$ and a spot size of $\sim 20 \mu \mathrm{m}$. A dual-wedge polarization scrambler was inserted to depolarize the laser. XPS data were collected using a Kratos AXIS Ultra spectrometer (Kratos Analytical, Manchester, U.K.). The instrument was equipped with a hybrid magnetic and electrostatic electron lens system, a delay-line detector, and a monochromatic $\mathrm{Al} \mathrm{K} \alpha \mathrm{X}$-ray source (1486.7 $\mathrm{eV})$. Data were collected at a pressure of $\sim 5 \times 10^{-9}$ Torr with photoelectrons collected at $0^{\circ}$ with respect to the sample. The analyzer pass energy was $80 \mathrm{eV}$ for the survey spectrum and 10 $\mathrm{eV}$ for all other spectra. The instrument energy scale and work 
function were calibrated using clean $\mathrm{Au}, \mathrm{Ag}$, and $\mathrm{Cu}$ standards. The instrument was operated by Vision Manager software v. 2.2.10 revision 5. The data were analyzed using CasaXPS software (CASA Software Ltd). XRD data were collected in a PANalytical X'Pert Pro X-ray powder diffractometer using the $\mathrm{Cu} K \alpha 1$ line $(\lambda=1.5406 \AA)$ with a tube voltage and current of $40 \mathrm{kV}$ and $20 \mathrm{~mA}$, respectively.

Coin Cell Preparation. Supercapacitor working electrodes consisted of GNSP material and polyvinylidene fluoride (PVDF, MTI Corporation, $\geq 99.5 \%$ ) binder in an 88:12 ratio and were mixed in $N$-methyl-2-pyrrolidinone (NMP, Sigma-Aldrich, 99.5\%) in a centrifugal mixer (AR-100 Thinky U.S.A., Inc., Laguna Hills, CA) at $5000 \mathrm{rpm}$ for $10 \mathrm{~min}$. A thin layer of the resulting slurry was spread across a stainless-steel spacer (MTI Corporation) with a spatula and dried at $120{ }^{\circ} \mathrm{C}$ in vacuum for $16 \mathrm{~h}$. Two-electrode 2032-coin cells were assembled in an argon-filled glovebox $\left(\mathrm{O}_{2}\right.$ : $<0.1 \mathrm{ppm}, \mathrm{H}_{2} \mathrm{O}$ : < $0.1 \mathrm{ppm})$. The counter/reference electrode was lithium foil (Sigma-Aldrich, 99.9\%, $0.75 \mathrm{~mm}$, mechanically cleansed immediately before cell assembly), and the separator was a propylene separator (Celgard 2400). The electrolyte was $1 \mathrm{M}$ $\mathrm{LiPF}_{6}$ in propylene carbonate/dimethyl carbonate (1:1 mixture by volume, both Sigma-Aldrich, $\geq 99 \%$, stored over molecular sieves, $3 \AA$, Beantown Chemical), and about eight drops of electrolyte were used in each coin cell. The electrolyte was mixed in a dried HDPE bottle.

Electrochemical Characterization. All electrochemical measurements were performed on a Reference 600 (Gamry Instruments, Warminster, PA). Capacitance was measured by cyclic voltammetry between 2 and $4 \mathrm{~V}$ at scan rates of 3.16, 10, $31.6,100,316$, and $1000 \mathrm{mV} / \mathrm{s}$. Electrochemical impedance spectroscopy measurements were taken potentiostatically at 2 $\mathrm{V}$ with an rms voltage of $\pm 5 \mathrm{mV}$. All voltages are with respect to $\mathrm{Li} / \mathrm{Li}^{+}$.

Electrochemical Exfoliation. Three in situ exfoliation procedures were investigated as follows. (I) The voltage of the graphene electrode was ramped from open-circuit potential $(\sim 2.7 \mathrm{~V})$ to $1 \mathrm{~V}$ at a rate of $1 \mathrm{mV} / \mathrm{s}$, held at $1 \mathrm{~V}$ for $3 \mathrm{~h}$, ramped to a high voltage at a rate of $1 \mathrm{mV} / \mathrm{s}$, held at the high voltage for $3 \mathrm{~h}$, then ramped to $2 \mathrm{~V}$ at a rate of $1 \mathrm{mV} / \mathrm{s}$. Then, the cyclic voltammetry and electrochemical impedance spectroscopy measurements were performed at $2 \mathrm{~V}$. This process was repeated for one cell such that the high voltage on the first cycle was $5 \mathrm{~V}$, then the high voltage on the second cycle was 6 $\mathrm{V}$, as so forth until the high voltage on the last cycle was $10 \mathrm{~V}$. (II) The voltage of the graphene electrode was ramped from open-circuit potential to $1 \mathrm{~V}$ at a rate of $10 \mathrm{mV} / \mathrm{s}$, held at $1 \mathrm{~V}$ for $1 \mathrm{~h}$, ramped to $6 \mathrm{~V}$ at a rate of $10 \mathrm{mV} / \mathrm{s}$, held at $6 \mathrm{~V}$ for $1 \mathrm{~h}$, then ramped to $2 \mathrm{~V}$ at a rate of $10 \mathrm{mV} / \mathrm{s}$, then the capacitance was measured by cycled voltammetry. This process was repeated 20 times. (III) The voltage of the graphene electrode was ramped from open-circuit potential to $1 \mathrm{~V}$ at a rate of 10 $\mathrm{mV} / \mathrm{s}$, held at $1 \mathrm{~V}$ for $1 \mathrm{~h}$, ramped to $6 \mathrm{~V}$ at a rate of $10 \mathrm{mV} / \mathrm{s}$, held at $10 \mathrm{~V}$ for $1 \mathrm{~h}$, then ramped to $2 \mathrm{~V}$ at a rate of $10 \mathrm{mV} / \mathrm{s}$, then the capacitance was measured by cycled voltammetry. This process was repeated 20 times. All voltages are with respect to $\mathrm{Li} / \mathrm{Li}^{+}$.

\section{ASSOCIATED CONTENT}

\section{SI Supporting Information}

The Supporting Information is available free of charge at https://pubs.acs.org/doi/10.1021/acsomega.0c06048.
Lithium intercalation in graphene; capacitance and enhancement of GNSP electrodes exfoliated without the $1 \mathrm{~V}$ exfoliation step; cyclic voltammogram capacitance measurements of GNSPs exfoliated with high voltages ranging from 5 to $10 \mathrm{~V}$ (Note 1 and Figure S1); cyclic voltammograms for graphene electrodes exfoliated at $5,6,7,8$, and $9 \mathrm{~V}$, as well as the reducedscale cyclic voltammograms of all electrodes (Figures S2-S9); and extrapolation of double-layer capacitance and solution resistance from cyclic voltammograms (Note 2 and Figure S10) (PDF)

\section{AUTHOR INFORMATION}

\section{Corresponding Author}

Nai-Chang Yeh - Department of Physics, California Institute of Technology, Pasadena, California 91125, United States; (1) orcid.org/0000-0002-1826-419X; Email: ncyeh@ caltech.edu

\section{Authors}

Jacob D. Bagley - Division of Chemistry and Chemical Engineering, California Institute of Technology, Pasadena, California 91125, United States

Dorte R. Danielsen - Department of Physics, California Institute of Technology, Pasadena, California 91125, United States; Department of Physics, Technical University of Denmark, Lyngby 2800, Denmark

Complete contact information is available at:

https://pubs.acs.org/10.1021/acsomega.0c06048

\section{Author Contributions}

J.D.B. designed the experiment; fabricated the GNSP materials; carried out Raman spectroscopy, XPS, XRD, and electrochemical measurements; and participated in preparing the manuscript. D.R.D. carried out GNSP dispersion and SEM imaging. N.-C.Y. coordinated and oversaw the research project and participated in preparing the manuscript.

\section{Funding}

This research was jointly supported by the United Advanced Materials (Award \#NCY.00012T15-1-GIFT.YEH0001T15) and the National Science Foundation under the Institute for Quantum Information and Matter (IQIM) at Caltech (Award \#1733907).

\section{Notes}

The authors declare no competing financial interest.

\section{ACKNOWLEDGMENTS}

The authors thank Professor George R. Rossman for allowing access to his Raman spectroscopy facilities, Professor Kimberly A. See for access to coin cell fabrication facilities, and the Beckman Institute at Caltech for access to XPS and electrochemical testing facilities.

\section{REFERENCES}

(1) Liu, C.; Yu, Z.; Neff, D.; Zhamu, A.; Jang, B. Z. Graphene-Based Supercapacitor with an Ultrahigh Energy Density. Nano Lett. 2010, 10, 4863-4868.

(2) Wu, Z.-S.; Winter, A.; Chen, L.; Sun, Y.; Turchanin, A.; Feng, X.; Müllen, K. Three-Dimensional Nitrogen and Boron Co-Doped Graphene for High-Performance All-Solid-State Supercapacitors. Adv. Mater. 2012, 24, 5130-5135.

(3) Zhu, Y.; Murali, S.; Stoller, M. D.; Ganesh, K. J.; Cai, W.; Ferreira, P. J.; Pirkle, A.; Wallace, R. M.; Cychosz, K. A.; Thommes, 
M.; Su, D.; Stach, E. A.; Ruoff, R. S. Carbon-Based Supercapacitors

Produced by Activation of Graphene. Science 2011, 332, 1537-1541.

(4) Chen, S.; Zhu, J.; Wu, X.; Han, Q.; Wang, X. Graphene Oxide$\mathrm{MnO} 2$ Nanocomposites for Supercapacitors. ACS Nano 2010, 4, $2822-2830$

(5) Geim, A. K. Graphene: Status and Prospects. Science 2009, 324, $1530-1534$

(6) Xia, J.; Chen, F.; Li, J.; Tao, N. Measurement of the Quantum Capacitance of Graphene. Nat. Nanotechnol. 2009, 4, 505-509.

(7) Li, Y.; Henzie, J.; Park, T.; Wang, J.; Young, C.; Xie, H.; Yi, J. W.; Li, J.; Kim, M.; Kim, J.; Yamauchi, Y.; Na, J. Fabrication of Flexible Microsupercapacitors with Binder-Free ZIF-8 Derived Carbon Films via Electrophoretic Deposition. Bull. Chem. Soc. Jpn. 2019, 93, 176181.

(8) Yoon, Y.; Lee, K.; Baik, C.; Yoo, H.; Min, M.; Park, Y.; Lee, S. M.; Lee, H. Anti-Solvent Derived Non-Stacked Reduced Graphene Oxide for High Performance Supercapacitors. Adv. Mater. 2013, 25, 4437-4444.

(9) Nagashio, K.; Nishimura, T.; Kita, K.; Toriumi, A. Mobility Variations in Mono- and Multi-Layer Graphene Films. Appl. Phys. Express 2009, 2, No. 025003.

(10) Tang, J.; Salunkhe, R. R.; Zhang, H.; Malgras, V.; Ahamad, T.; Alshehri, S. M.; Kobayashi, N.; Tominaka, S.; Ide, Y.; Kim, J. H.; Yamauchi, Y. Bimetallic Metal-Organic Frameworks for Controlled Catalytic Graphitization of Nanoporous Carbons. Sci. Rep. 2016, 6, No. 30295.

(11) Cataldo, F.; Compagnini, G.; Patané, G.; Ursini, O.; Angelini, G.; Ribic, P. R.; Margaritondo, G.; Cricenti, A.; Palleschi, G.; Valentini, F. Graphene Nanoribbons Produced by the Oxidative Unzipping of Single-Wall Carbon Nanotubes. Carbon 2010, 48, 2596-2602.

(12) Li, D.; Müller, M. B.; Gilje, S.; Kaner, R. B.; Wallace, G. G. Processable Aqueous Dispersions of Graphene Nanosheets. Nat. Nanotechnol. 2008, 3, 101-105.

(13) Hernandez, Y.; Nicolosi, V.; Lotya, M.; Blighe, F. M.; Sun, Z.; De, S.; McGovern, I. T.; Holland, B.; Byrne, M.; Gun'Ko, Y. K.; Boland, J. J.; Niraj, P.; Duesberg, G.; Krishnamurthy, S.; Goodhue, R.; Hutchison, J.; Scardaci, V.; Ferrari, A. C.; Coleman, J. N. High-Yield Production of Graphene by Liquid-Phase Exfoliation of Graphite. Nat. Nanotechnol. 2008, 3, 563-568.

(14) Wang, J.; Manga, K. K.; Bao, Q.; Loh, K. P. High-Yield Synthesis of Few-Layer Graphene Flakes through Electrochemical Expansion of Graphite in Propylene Carbonate Electrolyte. J. Am. Chem. Soc. 2011, 133, 8888-8891.

(15) Parvez, K.; Wu, Z.-S.; Li, R.; Liu, X.; Graf, R.; Feng, X.; Müllen, K. Exfoliation of Graphite into Graphene in Aqueous Solutions of Inorganic Salts. J. Am. Chem. Soc. 2014, 136, 6083-6091.

(16) Wei, D.; Grande, L.; Chundi, V.; White, R.; Bower, C.; Andrew, P.; Ryhänen, T. Graphene from Electrochemical Exfoliation and Its Direct Applications in Enhanced Energy Storage Devices. Chem. Commun. 2012, 48, 1239-1241.

(17) Parvez, K.; Li, R.; Puniredd, S. R.; Hernandez, Y.; Hinkel, F.; Wang, S.; Feng, X.; Müllen, K. Electrochemically Exfoliated Graphene as Solution-Processable, Highly Conductive Electrodes for Organic Electronics. ACS Nano 2013, 7, 3598-3606.

(18) Liu, Z.; Wu, Z.-S.; Yang, S.; Dong, R.; Feng, X.; Müllen, K. Ultraflexible In-Plane Micro-Supercapacitors by Direct Printing of Solution-Processable Electrochemically Exfoliated Graphene. Adv. Mater. 2016, 28, 2217-2222.

(19) Liu, Z.; Parvez, K.; Li, R.; Dong, R.; Feng, X.; Müllen, K. Transparent Conductive Electrodes from Graphene/PEDOT:PSS Hybrid Inks for Ultrathin Organic Photodetectors. Adv. Mater. 2015, $27,669-675$.

(20) Liu, X.; Ma, T.; Pinna, N.; Zhang, J. Two-Dimensional Nanostructured Materials for Gas Sensing. Adv. Funct. Mater. 2017, 27, No. 1702168.

(21) Märkle, W.; Tran, N.; Goers, D.; Spahr, M. E.; Novák, P. The Influence of Electrolyte and Graphite Type on the PF6- Intercalation Behaviour at High Potentials. Carbon 2009, 47, 2727-2732.
(22) Winter, M.; Besenhard, J. O.; Spahr, M. E.; Novák, P. Insertion Electrode Materials for Rechargeable Lithium Batteries. Adv. Mater. 1998, 10, 725-763.

(23) Winter, M.; Wrodnigg, G. H.; Besenhard, J. O.; Biberacher, W.; Novák, P. Dilatometric Investigations of Graphite Electrodes in Nonaqueous Lithium Battery Electrolytes. J. Electrochem. Soc. 2000, $147,2427$.

(24) Septiani, N. L. W.; Kaneti, Y. V.; Fathoni, K. B.; Wang, J.; Ide, Y.; Yuliarto, B.; Nugraha; Dipojono, H. K.; Nanjundan, A. K.; Golberg, D.; Bando, Y.; Yamauchi, Y. Self-Assembly of Nickel Phosphate-Based Nanotubes into Two-Dimensional Crumpled Sheetlike Architectures for High-Performance Asymmetric Supercapacitors. Nano Energy 2020, 67, No. 104270.

(25) Makino, S.; Yamauchi, Y.; Sugimoto, W. Synthesis of ElectroDeposited Ordered Mesoporous RuOx Using Lyotropic Liquid Crystal and Application toward Micro-Supercapacitors. J. Power Sources 2013, 227, 153-160.

(26) Hsu, C.-C.; Bagley, J. D.; Teague, M. L.; Tseng, W.-S.; Yang, K. L.; Zhang, Y.; Li, Y.; Li, Y.; Tour, J. M.; Yeh, N.-C. High-Yield SingleStep Catalytic Growth of Graphene Nanostripes by Plasma Enhanced Chemical Vapor Deposition. Carbon 2018, 129, 527-536.

(27) Bagley, J. D.; Kishore Kumar, D.; See, K. A.; Yeh, N.-C. Selective Formation of Pyridinic-Type Nitrogen-Doped Graphene and Its Application in Lithium-Ion Battery Anodes. RSC Adv. 2020, 10, 39562-39571.

(28) Yeh, N.-C.; Hsu, C.-C.; Bagley, J.; Tseng, W.-S. Single-Step Growth of Graphene and Graphene-Based Nanostructures by PlasmaEnhanced Chemical Vapor Deposition. Nanotechnology 2019, 30, No. 162001.

(29) Bo, Z.; Yang, Y.; Chen, J.; Yu, K.; Yan, J.; Cen, K. PlasmaEnhanced Chemical Vapor Deposition Synthesis of Vertically Oriented Graphene Nanosheets. Nanoscale 2013, 5, 5180-5204.

(30) Xu, W.; Lee, T.-W. Recent Progress in Fabrication Techniques of Graphene Nanoribbons. Mater. Horiz. 2016, 3, 186-207.

(31) Ferrari, A. C.; Basko, D. M. Raman Spectroscopy as a Versatile Tool for Studying the Properties of Graphene. Nat. Nanotechnol. 2013, 8, 235-246.

(32) Ferrari, A. C.; Robertson, J. Interpretation of Raman Spectra of Disordered and Amorphous Carbon. Phys. Rev. B 2000, 61, 1409514107.

(33) Yang, D.-Q.; Sacher, E. Carbon 1s X-Ray Photoemission Line Shape Analysis of Highly Oriented Pyrolytic Graphite: The Influence of Structural Damage on Peak Asymmetry. Langmuir 2006, 22, 860862.

(34) Blume, R.; Rosenthal, D.; Tessonnier, J.-P.; Li, H.; KnopGericke, A.; Schlögl, R. Characterizing Graphitic Carbon with X-ray Photoelectron Spectroscopy: A Step-by-Step Approach. ChemCatChem 2015, 7, 2871-2881.

(35) Kovtun, A.; Jones, D.; Dell'Elce, S.; Treossi, E.; Liscio, A.; Palermo, V. Accurate Chemical Analysis of Oxygenated GrapheneBased Materials Using X-Ray Photoelectron Spectroscopy. Carbon 2019, 143, 268-275.

(36) Major, G. H.; Shah, D.; Avval, T. G.; Fernandez, V.; Fairley, N.; Linford, M. R. Advanced Line Shapes in X-Ray Photoelectron Spectroscopy II.The Finite Lorentzian (LF) Line Shape (with Some MATLAB Code Illustrating the Use of the Subplot Function). Vac. Technol. Coat. 2020, 35-39.

(37) Peak Model for Highly Oriented Pyrolytic Graphite (HOPG) in CasaXPS; CasaXPS Casa Software, 2017. https://www.youtube. $\mathrm{com} /$ watch?v=PhsQ60jHzkU\&ab_channel=CasaXPSCasaSoftware.

(38) Díaz, J.; Paolicelli, G.; Ferrer, S.; Comin, F. Separation of the sp3 and sp2 Components in the C1s Photoemission Spectra of Amorphous Carbon Films. Phys. Rev. B 1996, 54, 8064-8069.

(39) Singh, B.; Hesse, R.; Linford, M. R. Good Practices for XPS (and Other Types of) Peak Fitting. Vac. Technol. Coat. 2015, 12, 2531.

(40) Darmstadt, H.; Roy, C. Surface Spectroscopic Study of Basic Sites on Carbon Blacks. Carbon 2003, 41, 2662-2665. 
(41) Conway, B. E. Electrochemical Supercapacitors. In Scientific Fundementals and Technological Applications, 1st ed.; Kluwer Academic/Plenum Publishers: New York, 1999; pp 525-556.

(42) Yoo, E.; Kim, J.; Hosono, E.; Zhou, H.; Kudo, T.; Honma, I. Large Reversible Li Storage of Graphene Nanosheet Families for Use in Rechargeable Lithium Ion Batteries. Nano Lett. 2008, 8, 22772282.

(43) Lian, P.; Zhu, X.; Liang, S.; Li, Z.; Yang, W.; Wang, H. Large Reversible Capacity of High Quality Graphene Sheets as an Anode Material for Lithium-Ion Batteries. Electrochim. Acta 2010, 55, 39093914.

(44) Seel, J. A.; Dahn, J. R. Electrochemical Intercalation of PF 6 into Graphite. J. Electrochem. Soc. 2000, 147, 892.

(45) Etacheri, V.; Marom, R.; Elazari, R.; Salitra, G.; Aurbach, D. Challenges in the Development of Advanced Li-Ion Batteries: A Review. Energy Environ. Sci. 2011, 4, 3243-3262.

(46) Murmann, P.; Schmitz, R.; Nowak, S.; Ignatiev, N.; Sartori, P.; Cekic-Laskovic, I.; Winter, M. Electrochemical Performance and Thermal Stability Studies of Two Lithium Sulfonyl Methide Salts in Lithium-Ion Battery Electrolytes. J. Electrochem. Soc. 2015, 162, A1738.

(47) Bard, A. J.; Faulkner, L. R. Electrochemical Methods: Fundamentals and Applications, 2nd ed.; Swain, E., Ed.; John Wiley \& Sons, Inc: New York, 2000; pp 14-18.

(48) Bisquert, J.; Compte, A. Theory of the Electrochemical Impedance of Anomalous Diffusion. J. Electroanal. Chem. 2001, 499, $112-120$.

(49) Sharifi-Viand, A.; Mahjani, M. G.; Jafarian, M. Investigation of Anomalous Diffusion and Multifractal Dimensions in Polypyrrole Film. J. Electroanal. Chem. 2012, 671, 51-57. 